\title{
Detection and Extraction of OFDM Parameters Using Difference of Gaussians
}

\author{
Amin Naemi ${ }^{\text {a,* }}$ \\ ${ }^{a}$ PhD Student, Maersk Mc-Kinney Møller Institute, Southern Denmark University,Denmark
}

Received: 18 March 2019; Accepted: 09 August 2019; Published: 08 September 2019

\begin{abstract}
Signals type detection is very important in telecommunication. Telecommunication signals can be divided into two major groups: single-carrier signals and multi-carrier signals. The first step in extracting data in multicarrier communication signals is to detect signals and their subcarriers. OFDM signals are one of the most popular multi-carrier signals that are used widely. This paper will introduce a blind detection method for OFDM signals, subcarriers, and the central frequency of them based on the Difference of Gaussians (DoG) technique which is applied for blob detection in machine vision. Performance of our method is compared with high-resolution spectral estimation such as Capon, Borgiotti-Lagunas, and MUSIC. Results showed that it has less computational complexity than the others. Also, there is no need to learn parameters, so the response time of the system is appropriate. Furthermore, many tests have been done on real and artificial signals corrupted with noise and fading and the results showed our proposed method has better performance and cause the lower error in the severe condition like SNR=0.
\end{abstract}

Index Terms: Multi-carrier signals, OFDM, difference of Gaussians, machine vision.

(C) 2019 Published by MECS Publisher. Selection and/or peer review under responsibility of the Research Association of Modern Education and Computer Science

\section{Introduction}

Orthogonal frequency-division multiplexing (OFDM) is a popular modulation scheme that has been widely adopted in wireless broadband systems to combat frequency-selective fading in wireless channels[1]. One of the most important components of the cognitive radio concept is the ability to measure, sense, learn, and be aware of the parameters related to the radio channel characteristics, availability of spectrum and power, radio operating environment, user requirements and applications, available networks (infrastructures) and nodes, * Corresponding author.

E-mail address: amin@mmmi.sdu.dk 
local policies, and other operating restrictions[2].

Nowadays, multi-carrier and single-carrier signals are used for sending data in digital telecommunication that each of them has advantages and disadvantages. Generally, using multicarrier signals to send data have been increased in recent years. For example, when the channel is unreliable due to fading in short periods, only some subcarriers can be used to send data and the receiver can extract the real data from received data of same subcarriers used in the transmitter. However, multicarrier signals have some deficiencies such as difficulty in accurate determination of signal central frequencies due to the presence of noise and fading. additionally, this type of telecommunication has some problems such as symbol and subcarrier interference.

OFDM signals are made up of many overlapping subcarriers. Central frequency is constant between two subcarriers and the structure of subcarriers is almost Gaussian[3]. In this paper, we used this feature of subcarriers (Gaussians form) to detect OFDM signals subcarriers by using the difference of Gaussian technique. The proposed method has four steps. First, pre-process is done on the signal's spectrum to make it smoother, second the spectrum is reprocessed, then the convolution is calculated by DoG for different standard deviation values and finally, the standard deviation corresponding to the highest amount of energy is selected, then we can detect subcarriers and their central frequencies using optimal standard deviation.

This paper is structured as follows: In Section 2, the literature review is included. Section 3 shows OFDM signal can be modeled as a Gaussian distribution. In Section 4, the proposed method is described in detail. Experiments are described in Section 5 and conclusions are presented in Section 6.

\section{Related Works}

OFDM signals as a multicarrier signal are widely used in urban and military applications because of efficient bandwidth using and robustness against fading. Therefore, it is essential to introduce a blind method for signal type detection and parameters extraction. There are many papers on the detection and extraction of OFDM signals parameters such as carrier's recovery, carrier frequency offset, etc. [4-8]. One of the common methods is based on cyclostationary that have good performance in many applications. This technique is used for signal detection[9, 10], channel estimation[11] and time and frequency[12] synchronization, however; it is not suitable for military applications due to high computational complexity and time-consuming.

Traditional methods for spectral estimation work based on Fourier transform. In this approach, a window with a specific length is used to decrease the effects of side lobes. In this case, the resolution of the signal is $1 / \mathrm{M}$ where $\mathrm{M}$ is the number of samples located in each section related to the size of the window. Despite these methods, high-resolution methods do not use Fourier transform to estimate the spectrum of the signal. They use the stationary property of signal in short periods, so they can estimate the signal with a resolution higher than 1/M[13]. In this section, we introduce three high-resolution spectral estimations briefly and since they have high accuracy we compare our proposed method with them.

In high-resolution methods, the goal is estimating the power of a signal in different frequencies regardless of the energy of other frequencies. Capon use frequency-selective filter for this purpose[13]. The equation (1) Shows the estimated power spectrum of the signal in the frequency of $f_{k}$.

$$
\hat{R}_{M}\left(e^{j 2 \pi f_{k}}\right)=E\left\{\left|y_{k}(n)\right|^{2}\right\}=\frac{M}{\mathrm{v}^{H}\left(f_{k}\right) \mathrm{R}_{x}^{-1} \mathrm{v}\left(f_{k}\right)}
$$

Where $y_{k}$ is the output of the kth filter, $V(f)=\left[1 e^{-j 2 \pi f} \ldots e^{-j 2 \pi f(M-1)}\right]^{T}, \mathrm{R}_{\mathrm{x}}=E\left\{\mathrm{x}(n) \mathrm{x}^{\mathrm{H}}(n)\right\}$ and $\mathrm{H}$ is Hermitian transform. In Borgiotti-Lagunas, estimated power calculated in this method is shown in equation (2).

$$
\widehat{P}\left(f_{k}\right)=\frac{v^{H}\left(f_{k}\right) R_{x}^{-1} v\left(f_{k}\right)}{v^{H}\left(f_{k}\right) R_{x}^{-2} v\left(f_{k}\right)}
$$


There is also another approach called MUSIC. In this approach, the signal is divided into two sections: signal and noise. The signal consists of $\mathrm{p}$ exponential components and the correlation matrix should be computed. So $\mathrm{p}$ eigenvalues determine the original signal and M-p eigenvalues define noise[14]. The predicted power in this method is shown in equation (3).

$$
\hat{P}\left(f_{k}\right)=\frac{1}{\sum_{i=p+1}^{M}\left|V^{H} q_{i}\right|^{2}}
$$

Where $q_{i}$ is eigenvectors of noise and $V$ is defined in the previous section.

\section{Modeling OFDM Signal as a Gaussian Distribution}

OFDM signal in baseband is shown in equation (4).

$$
s(t)=\sum_{i=0}^{N-1} A_{i} e^{\mathrm{j}\left(2 \pi f_{i} t+\phi_{i}\right)}
$$

Where $A_{i}, f_{i}$, and $\phi_{i}$ are amplitude, frequency, and phase of $i^{\text {th }}$ subcarrier respectively and $\mathrm{N}$ is the number of the subcarriers. The spectrum of an OFDM signal with five subcarriers is shown in Fig.1.

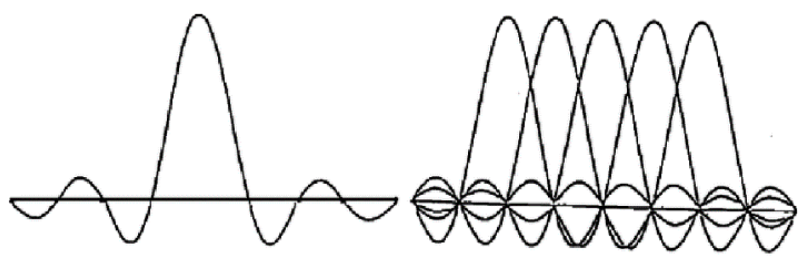

(a)

(b)

Fig.1. Spectrum of (a) OFDM subchannel and (b) OFDM signal overlapping subcarriers [15]

Based on the Fig.1, it seems that every subcarrier of the OFDM signal is like Gaussian distribution. Many researchers have proposed different approaches to model subcarriers of the OFDM signals with Gaussian distribution. Ehm et al. [16] have proved that an OFDM signal can be modeled using Gaussian distribution Since an OFDM signal is a linear superposition of stochastic independent random variables - the modulated subcarriers - the superposition obeys the central limit theorem. This observation leads to the assumption that the time domain OFDM signal can be accurately modeled by a mean-free normal distributed Gaussian random process with probability density function as equation (5).

$$
p_{x}(x)=\frac{1}{\sqrt{2 \pi \sigma_{i}^{2}}} \exp \left(-\frac{x^{2}}{2 \sigma_{i}^{2}}\right)
$$

Wei et al. [17] have shown that under some conditions, as the number of subcarriers goes to infinity, the continuous-time domain OFDM signal distribution converges to correlated complex Gaussian distribution. Kotzer et al. [18] have introduced a normalized Gaussian distribution to model OFDM signal and claimed that their model gives much better results than the conventional Gaussian model in the approximation of statistical properties of an OFDM signal. 


\section{Proposed Method}

In this section, different parts of our proposed method (SR-DoG) for detection of OFDM parameters are presented. Firstly, a preprocessing is done on the signal. In this step, an averaging filter is applied on the signal. In the next step, spectrum reprocessing of the signal is computed, then one of blob detection methods named difference of Gaussians is used to calculate the number subcarriers and the last step is finding the central frequencies of subcarriers. These steps are shown in Fig.2.

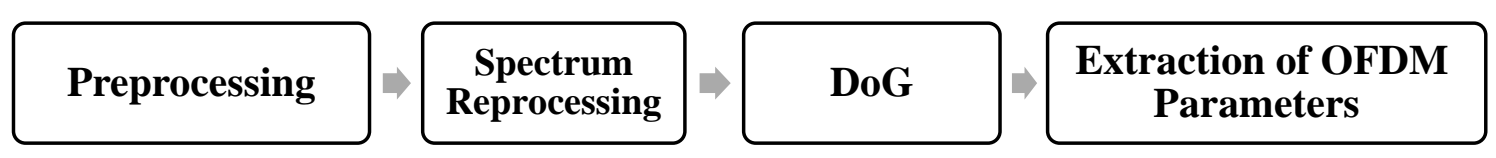

Fig.2. SR-DoG steps

\subsection{Preprocessing}

Initially, we preprocess a signal due to potential fast noise-like changes in the spectrum. This pre-processing includes using an averaging filter. Averaging filters have limited pulse response which is used to analyze the data with a different average for various subsets compared to whole data. Equation (6) shows an averaging filter.

$$
y(i)=\frac{1}{M} \sum_{j=0}^{M-1} x(i+j)
$$

Where $M$ is the length of the window. Since in OFDM signals the average of various samples of each subcarrier is different from the average of all spectrum samples, an averaging filter can be used for smoothing and increasing the output accuracy. It is crucial to choose the correct length for the window of the filter.

In OFDM signals, subcarriers have high overlapping and in real applications due to the presence of noise and fading some subcarriers can be weakened, so selecting the wrong length for windows of the filter can lead to deleting of some subcarriers and consequently the number of subcarriers may be considered wrong. Based on tests which were done on real signals, the right length of the window is calculated to equal to the number of samples between two consecutive peaks in the signal spectrum. For this purpose, the multiplication of the signal and window spectrum is computed, then the reverse Fourier transform is applied on the output. Equation (7) shows these stages.

$$
Y(t)=\operatorname{IFFT}(F F T(s(t)) \times F F T(W(t)))
$$

Where $s(t)$ and $W(t)$ are signal and window in the time domain.

\subsection{Spectrum Reprocessing}

Spectrum Reprocessing (SR) is the power spectrum of the power spectrum of the signal. This technique uses the quasi-period property of frequency domain and specifies the area of signal presence. SR can be calculated according to equation (8). 
$s\left(t^{\prime}\right)=\left|F F T\left[\left|F F T\left[S_{O F D M}(t)\right]\right|^{2}\right]\right|^{2}=\left|F F T\left[P_{O F D M}(\omega)\right]\right|^{2}$

Where

$$
S_{O F D M}(t)=\frac{1}{N_{S}} \sum_{n=1}^{N_{s}} A_{n}(t) e^{j\left[\omega_{n} t+\phi_{n}(t)\right]}
$$

Zhang et al showed that SR has more periodic properties than the power spectrum of the signal and have great ability to decrease the effect of noise or fading. For OFDM signal, its autocorrelation function contains narrow pulse periodically appearing in all points but the noise after spectrum reprocessing has gained only for zero points [3]. Therefore, spectrum reprocessing improves output SNR and decrease the effect of additive noise and has higher detecting capability.

\subsection{Difference of Gaussians}

The difference of Gaussians (DoG) is a method for blob detection. The blob detection in image processing and machine vision means the detection of points or areas of the image which have different features like lighting or color compared to their surroundings[19]. There are two major classes for blob detection algorithms based on the application: 1-differential methods: these methods work based on the derivative of the defined function. 2-Methods which are based on local extremum points: in these methods, minimum and maximum points of a defined function are computed.

DoG, as its name shows, is a differential method for blob detection. In this method, the differences of two Gaussians, regarding the different values for standard deviation, is calculated. The formula of DOG is shown in equation (10).

$$
D o G=\frac{1}{\sigma_{1} \sqrt{2 \pi}} \exp \left(-\frac{(x-\mu)^{2}}{2 \sigma_{1}^{2}}\right)-\frac{1}{\sigma_{2} \sqrt{2 \pi}} \exp \left(-\frac{(x-\mu)^{2}}{2 \sigma_{2}^{2}}\right)
$$

Where $\mu$ is the mean of Gaussians and $\sigma$ is the standard deviation. The difference of Gaussians function is shown in Fig.3.

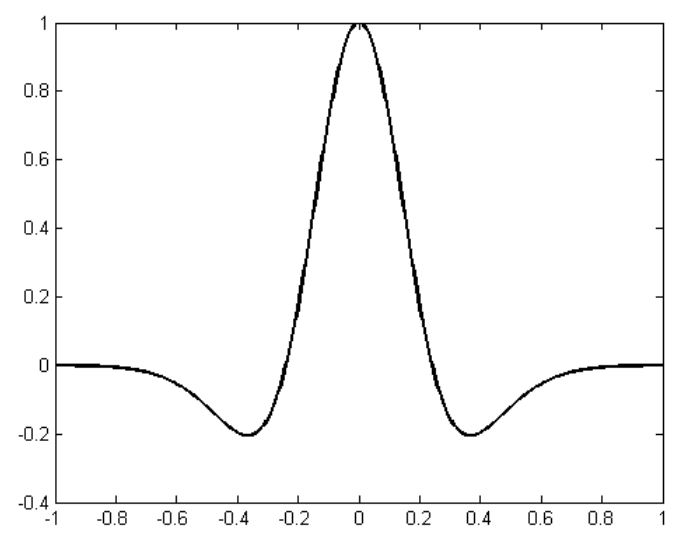

Fig.3. Difference of Gaussian function 
As we can see the DoG function is similar to OFDM subcarriers. As we discussed in section 2, subcarriers of an OFDM signal can be modeled using Gaussian distribution so, we can use different standard deviation to fit a DoG on the OFDM signal subcarriers.

The operation of the human brain for detection of subcarriers number in a spectrum is based on finding a repetitive pattern in the spectrum. It can be used for automatic detection of subcarriers number. Since the subcarriers are in Gaussians shape in OFDM signals, DoG can be used for subcarriers detection. For this purpose, two Gaussian kernels with different values for standard deviation are used. The convolution of the spectrum signal with differences of Gaussians and energy of output spectrum for different values of standard deviation was computed. The best value of standard deviation is related to the spectrum which has the greatest energy. experiments indicated that the greatest amount of energy corresponds to Gaussians width equals to subcarriers width. It means that by using this method Gaussian functions should be matched to subcarriers.

\subsection{Finding central frequency of subcarriers}

After detection of subcarriers, the central frequency of subcarriers should be computed. For this purpose, the center of Gaussians which are coincident with subcarriers is used. It should be noted, if the signal is demolished by noise or fading then the center of Gaussians may not be coincident with subcarriers and in this condition, the average of Gaussians center and the peak of subcarriers can be used as the central frequency.

\section{Experiments}

The experiments are divided into two categories: 1- experiments conducted on the real signals used in practical applications. 2-experiments done on artificial signals. The artificial data was produced to check the performance of the proposed method when the amount of destructive factors such as multi-path fading and white noise are known.

\subsection{Experiments on real data}

In this section, we study the performance of SR-DoG on real data. It is worth noting that no information was available for these signals. It means that there was not any prior information about the frequency range of signals. In this case, we could find the signal frequency range by using a threshold but another problem might appear and that was the existence of Doppler tone which caused the error in signal bandwidth detection. To resolve this problem, we considered a frequency range and checked the existence of a signal in it, thus, the start and end frequencies of a signal could be specified. The spectrum of the real OFDM signal is shown in Fig.4.

As can be seen in Fig.4, subcarriers are unidentifiable due to the presence of noise and fading. also, amplitudes of some subcarriers have been weakened. 


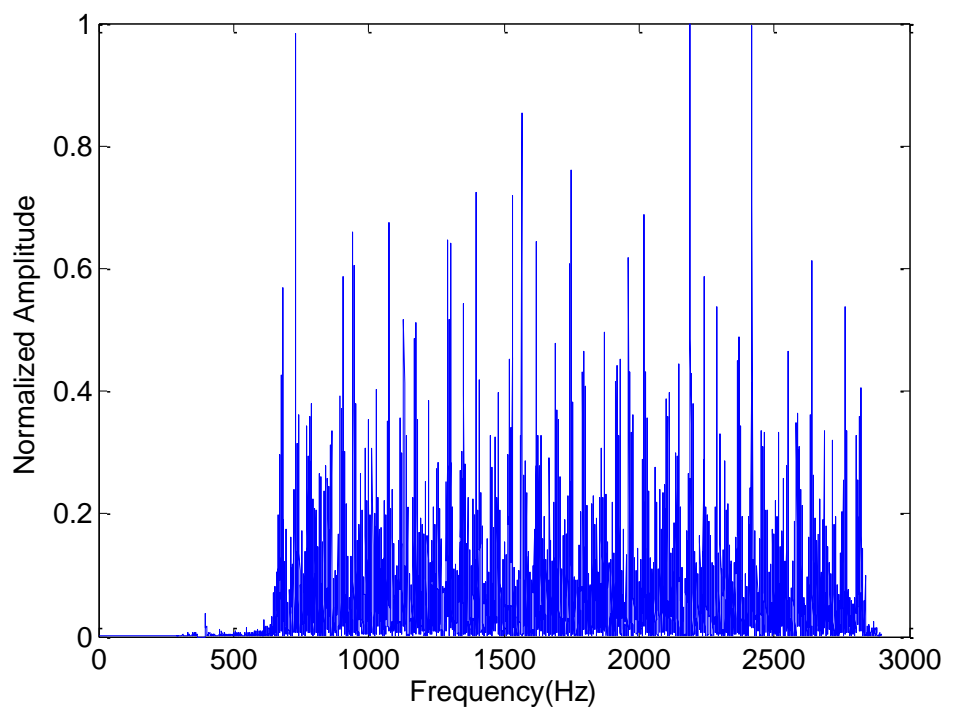

Fig.4. The spectrum of OFDM signals

As mentioned in previous sections, in the next step we smoothed the spectrum by using the averaging filter. The result is shown in Fig.5.

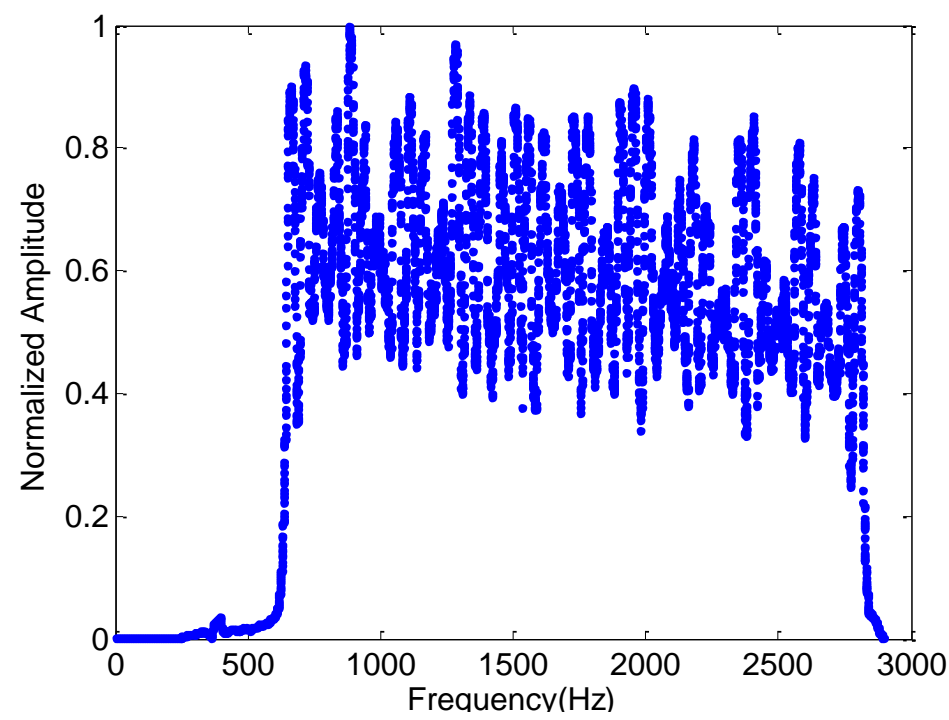

Fig.5. OFDM signal spectrum after preprocessing. 


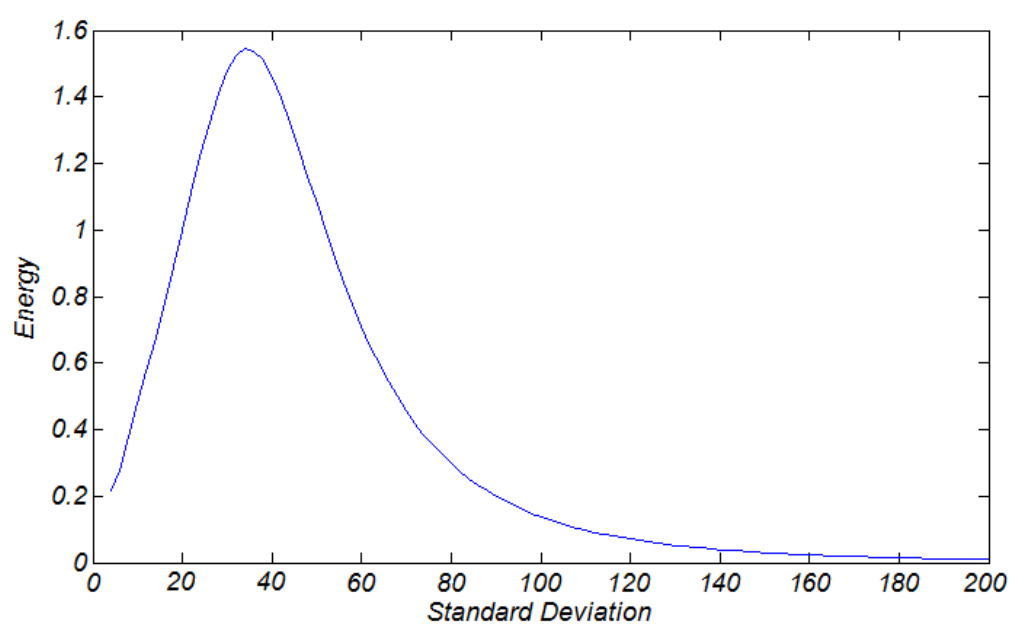

Fig.6. The energy of DoG according to different values for standard deviation.

It is obvious that in Fig.5, samples that existed as a single-peak are disappeared in this figure, so counting the number of subcarriers is much easier now, but the spectrum is not accurate enough and clears yet to determine the location of subcarriers. So, next the spectrum reprocessing of the signal should be computed and the best value of standard deviation for DOG should be determined. The amount of energy for different values of standard deviation is shown in Fig.6.

According to Fig.6, the appropriate standard deviation for this signal is about 40. By using this value and computing DoG and its convolution with spectrum reprocessing from the previous step, the final output of the system can be obtained that is shown in Fig.7. In this figure, the number of subcarriers was detected 39 correctly. Also, the central frequency of each subcarrier is marked with red color.

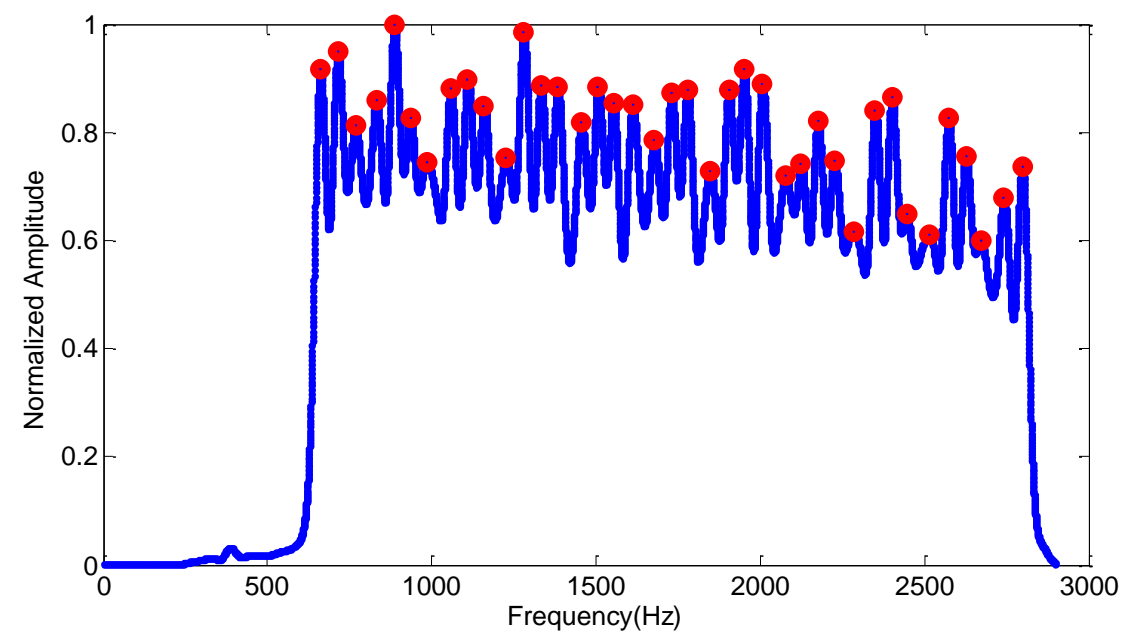

Fig.7. Subcarriers and their central frequency. 
SR-DoG compared with high-resolution method regard to the average of false detection. The result is shown in Fig.8. as it is seen all methods had great performance for SNR higher than four, but in lower SNRs especially in SNR=0, SR-DoG had better performance and cause the lower error.

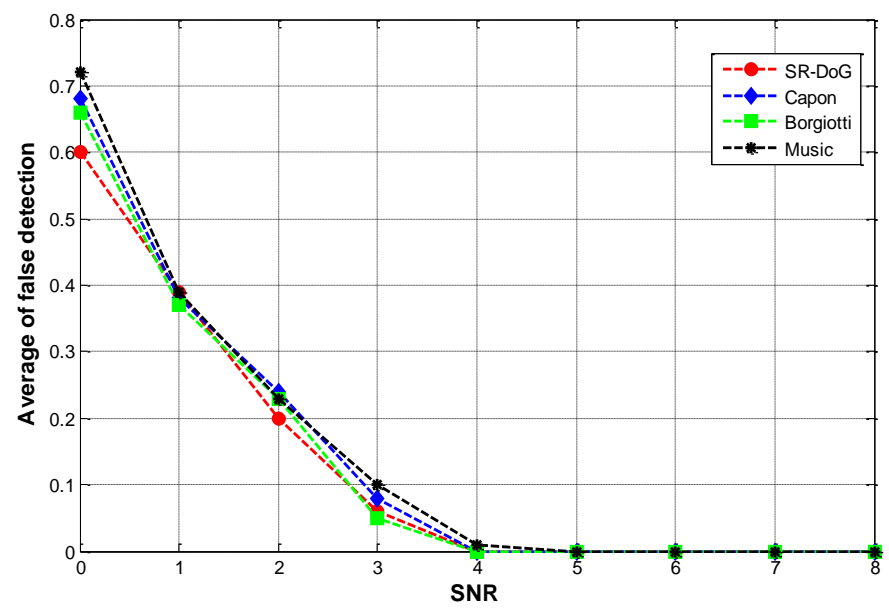

Fig.8. Performance of methods for real OFDM signals detection with 39 subcarriers.

It should be noted, the performance of the SR-DOG method was better than the other methods for real signals because in this approach we tried to find the optimal Gaussian to fit on subcarriers, so boosting of frequencies around the central frequency or weakening the central frequency which is causing error cannot affect the performance of this method. But in other methods, it is more likely that a wrong frequency- near the central frequency - was corrupted and boosted and selected as a central frequency.

\subsection{Experiments on artificial data}

In this section, the performance of different methods for extracting parameters of OFDM signals is evaluated. These methods include SR-DoG, Capon, Borgiotti-Lagunas, and music.

For this purpose, according to equation (4), the OFDM signals were produced, then the Gaussian noise and fading were added to data. The applied noise was mean zero Gaussians noise. Also, the multipath fading model was used for adding the effect of fading, which means that the weakened versions of the signal reached to the receiver with delay and interfered with the main signal. This type of fading in the wireless communication system is very common because the signal collides to the obstacles then reflects and finally gets to the destination. For simulation, the fading factors $0.1,0.4$ were used. The tests were repeated 50 times and the final error was computed by averaging of the number of subcarriers detection errors. Since by increasing the number of subcarriers, detection and extracting of the subcarriers become more difficult, the experiments were done for two types of OFDM artificial signals with 16 and 39 subcarriers under different SNRs. The performance of SRDoG compared with high-resolution spectral estimation.

The results of tests for real and artificial data with 39 subcarriers is shown in Fig.9 and Fig.10. 


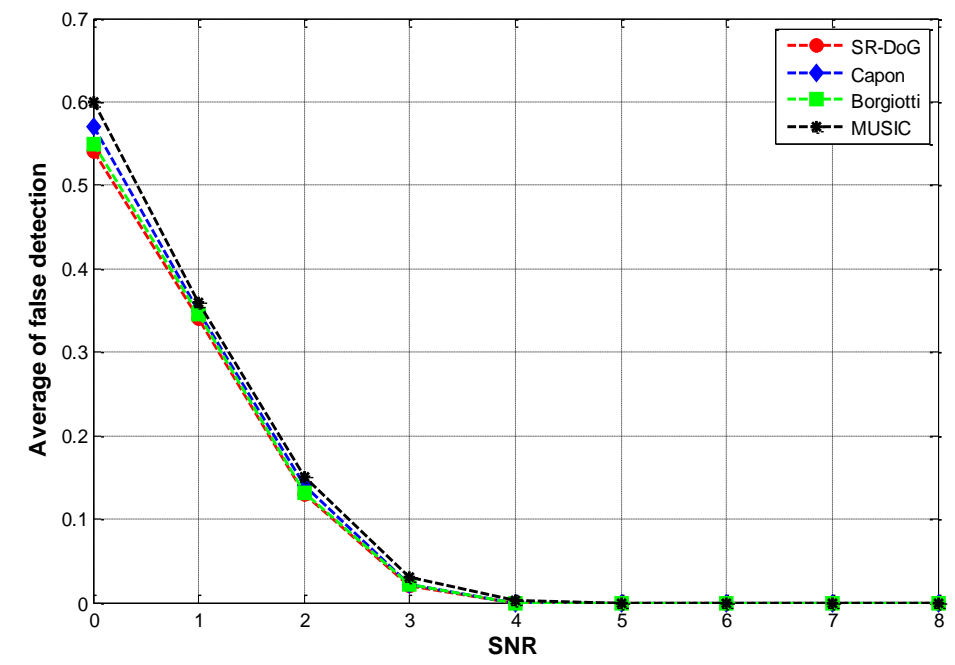

Fig.9. performance of methods for artificial OFDM signals detection with 39 subcarriers.

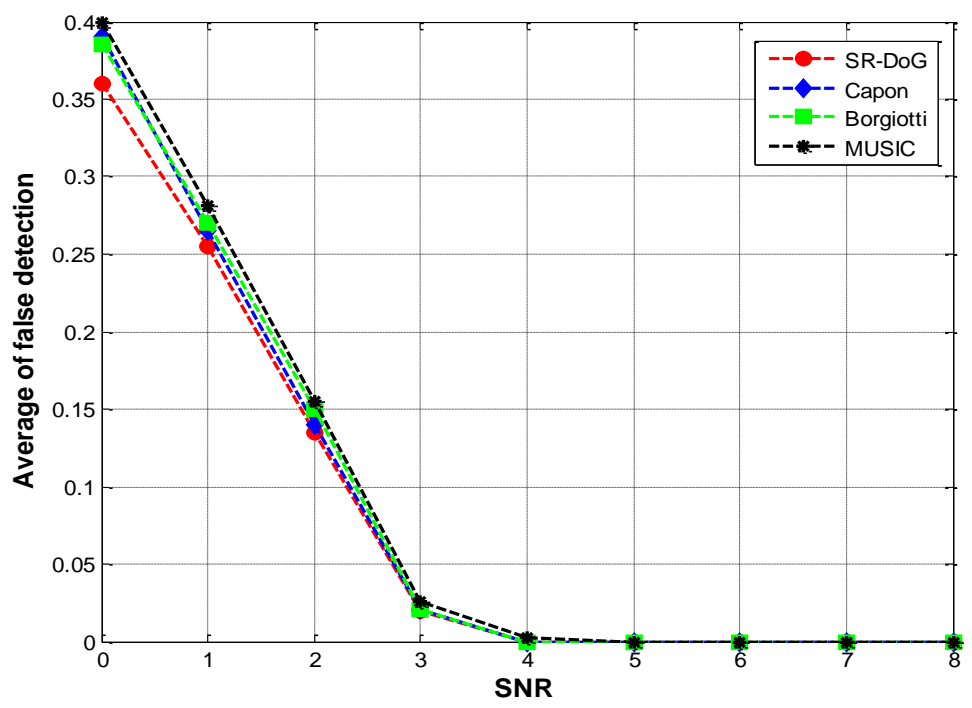

Fig.10. Performance of methods for artificial OFDM signals detection with 16 subcarriers

The SR-DOG made better result, especially for low SNRs. In general, all methods could detect the number of subcarriers correctly for SNRs higher than four.

\subsection{Execution time}

Since OFDM signals are used in different fields such as telecommunication and military applications, the execution speed and response time are very important. Therefore, in this section response time of different methods for the different number of samples under SNR=10 was investigated. For this goal, several artificial signals with the sample size of $8000,10000,20000,50000$ were selected, then different methods were used to 
extract parameters. The test was repeated 50 times and the average response time has been calculated by second. The result of this test was shown in Fig.11.

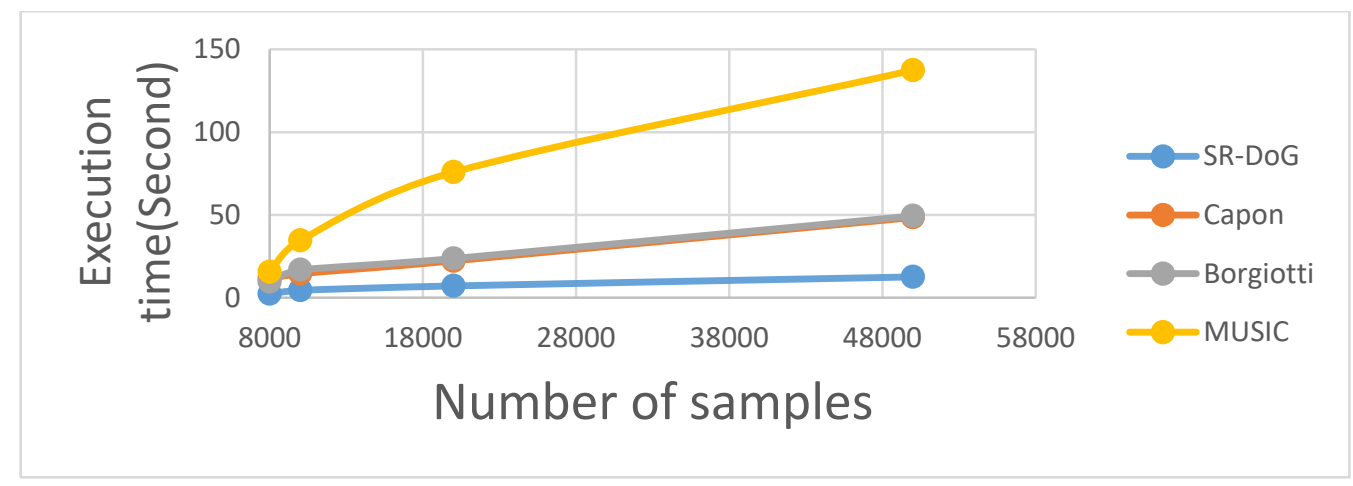

Fig.11. The response time of OFDM signal detection.

There are obvious differences between the response time of SR-DoG and the other methods. high-resolution methods are dependent on data so they need much more time than SR-DoG. In these approaches, the correlation matrix should be calculated so it takes much time but in SR-DoG the location of subcarriers and central frequencies could be calculated by the shape of the signal.

\section{Conclusions}

In this paper, a method for blind detection of OFDM signal and its parameter was introduced. This method has 4 stages including (i) preprocessing (ii)spectrum reprocessing (iii) using the difference of Gaussian(DoG) technique and (iii) subcarriers and their central frequencies detection. This method was applied for real data and artificial data. Real data had channel noise and different types of fading and in artificial data, the Gaussian noise and multipath fading were used. The performance of our method compared with high-resolution spectral estimation because these methods can estimate the spectrum of the signal very accurately. The results of tests were satisfying and we achieved better results than high-resolution methods especially in low SNRs. Also, the response time of our method was very lower than high-resolution methods, so it seems using SR-DoG in realtime applications is more practical in low SNRs.

\section{References}

[1] Ye, H., G.Y. Li, and B.-H.J.I.W.C.L. Juang, Power of deep learning for channel estimation and signal detection in OFDM systems. 2018. 7(1): p. 114-117.

[2] Sun, X., et al., A blind OFDM signal detection method based on cyclostationarity analysis. 2017. 94(3): p. 393-413.

[3] Zhang, H.-y. and C.-w. Yuan. A Method for Blind Detection of OFDM Signal Based on Power Spectrum Reprocessing. in Software Engineering, Artificial Intelligence, Networking, and Parallel/Distributed Computing, 2007. SNPD 2007. Eighth ACIS International Conference on. 2007. IEEE.

[4] Chen, Q., et al. Computation-efficient blind estimation of OFDM signal parameters for interception and data recovery. in SPIE Defense, Security, and Sensing. 2011. International Society for Optics and Photonics. 
[5] Chen, Q., et al. Progressive automatic detection of OFDM system parameters for universal mobile DTV receiver. in Vehicular Technology Conference Fall (VTC 2010-Fall), 2010 IEEE 72nd. 2010. IEEE.

[6] Le Nir, V., et al., Blind CP-OFDM and ZP-OFDM parameter estimation in frequency selective channels. EURASIP Journal on wireless communications and networking, 2009. 2009(1): p. 315765.

[7] Shi, M., Y. Bar-Ness, and W. Su. Blind OFDM systems parameters estimation for software defined radio. in New Frontiers in Dynamic Spectrum Access Networks, 2007. DySPAN 2007. 2nd IEEE International Symposium on. 2007. IEEE.

[8] Wang, R., et al., Channel estimation, carrier recovery, and data detection in the presence of phase noise in OFDM relay systems. IEEE Transactions on Wireless Communications, 2016. 15(2): p. 1186-1205.

[9] Prema, G. and P. Gayatri. Blind spectrum sensing method for OFDM signal detection in Cognitive Radio communications. in Communication and Network Technologies (ICCNT), 2014 International Conference on. 2014. IEEE.

[10] Sohn, S.H., et al. OFDM signal sensing method based on cyclostationary detection. in Cognitive Radio Oriented Wireless Networks and Communications, 2007. CrownCom 2007. 2nd International Conference on. 2007. IEEE.

[11] Bolcskei, H., Blind estimation of symbol timing and carrier frequency offset in wireless OFDM systems. IEEE Transactions on Communications, 2001. 49(6): p. 988-999.

[12] Heath, R.W. and G.B. Giannakis, Exploiting input cyclostationarity for blind channel identification in OFDM systems. IEEE Transactions on Signal Processing, 1999. 47(3): p. 848-856.

[13] Manolakis, D.G., V.K. Ingle, and S.M. Kogon, Statistical and adaptive signal processing: spectral estimation, signal modeling, adaptive filtering, and array processing. Vol. 46. 2005: Artech House Norwood.

[14] Hayes, M.H., Statistical digital signal processing and modeling. 2009: John Wiley \& Sons.

[15] Ghosh, S.J.a.p.a., Performance evaluation on the basis of Bit error rate for different order of Modulation and different length of Subchannels in ofdm system. 2014.

[16] Ehm, H., S. Winter, and R. Weigel. Analytic quantization modeling of OFDM signals using normal Gaussian distribution. in 2006 Asia-Pacific Microwave Conference. 2006. IEEE.

[17] Wei, S., D.L. Goeckel, and P.A.J.I.T.o.I.T. Kelly, Convergence of the complex envelope of bandlimited OFDM signals. 2010. 56(10): p. 4893-4904.

[18] Kotzer, I., et al. A model for OFDM signals with applications. in European Wireless 2012; 18th European Wireless Conference 2012. 2012. VDE.

[19] Bretzner, L. and T. Lindeberg, Feature tracking with automatic selection of spatial scales. Computer Vision and Image Understanding, 1998. 71(3): p. 385-392.

\section{Author's Profile}

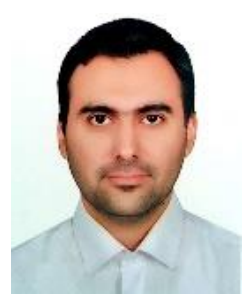

Amin Naemi is currently a Ph.D. student at the University of Southern Denmark. He received his M.Sc. in Artificial Intelligence from Amirkabir University (Tehran Polytechnics) and his B.Sc. in Computer Hardware Engineering from K.N Toosi University of Technology from Iran. 
How to cite this paper: Amin Naemi, "Detection and Extraction of OFDM Parameters Using Difference of Gaussians", International Journal of Wireless and Microwave Technologies(IJWMT), Vol.9, No.5, pp. 12-24, 2019.DOI: $10.5815 / \mathrm{ijwmt} .2019 .05 .02$ 